dienkunde, Mediennutzung, Medienkritik und Mediengestaltung erstreckt. Die Forderung nach Einführung eines neuen Unterrichtsfaches in vielen Bildungsinstitutionen, das diese wichtigen Gebiete abdecken soll, ergab sich in der Diskussion zwangsläufig.

Bei all diesen Varianten von Rationalität und Rationalitätsvorstellungen, ihren Wandlungen und Herausforderungen ist es für Wissenschaftler und Philosophen letztlich doch entscheidend, sich auf die wesentlichen, gemeinsamen Charakteristika zu besinnen. Daher sei am Ende des Berichts noch besonders das Plenarreferat von Professor Dr. Wiesław Sztumski (Universität Katowice) hervorgehoben, der sich die Frage stellte: „Wozu Rationalität?“. Er legte eine klare Begriffsbestimmung vor, nach der Rationalität auf dem Kausalprinzip beruhe und folgende Merkmale aufweise: Sinnfälligkeit, Zweckmäßigkeit, Rentabilität sowie Vorhersagbarkeit. Im Hinblick auf das Ziel der Menschheit, das fundamental sei und jedes andere Ziel erst rational begründbar mache, kritisierte er [zu Recht!] das globale Ziel unserer Zeit ,Sich bereichern und überleben!“, mit dem das Haben vor das Sein gestellt werde. Er forderte die Anerkennung des Guten, die alle Formen von Rationalität zu echter Vernünftigkeit emporhebe und der rationalisierenden Relativität entgegenwirke.

Nach dieser intensiven und anregenden Orientierung im Bereich der Rationalität wird von den Organisatoren daran gedacht, eine jährliche Rationalitätskonferenz mit wechselnden Schwerpunkten zu organisieren. Für den Herbst 2003 wird der Themenkreis ,,Rationalität und Ethik" ins Auge gefasst. Nähere Informationen sind zu erhalten am Institut für Philosophie, Schlesische Universität, PL 40007 Katowice; E-Mail: noras@ @aba.wns.us.edu.pl

\section{NRW im Dickicht der Nachhal- tigkeits-Indikatoren. Wie misst man Nachhaltigkeit?}

KöIn, 7. September 2001

\section{Tagungsbericht von Volker Stelzer, ITAS}

Der Workshop „NRW im Dickicht der Nachhaltigkeits-Indikatoren. Wie misst man Nachhaltigkei?" fand am 07. September 2001 an der Universität Köln statt. Anlass war der gemeinsame Wunsch unterschiedlicher Organisationen und Personen, die sich wissenschaftlich und praktisch mit dem Thema Nachhaltigkeitsindikatoren beschäftigen, nach einem breiten Austausch über die Stärken und Schwächen der von ihnen entwickelten unterschiedlichen Ansätze zur Indikatorenbildung. Zu diesem Zweck hatten Sie zu dem Workshop knapp 80 Experten aus Wissenschaft und Praxis eingeladen, mit denen die Indikatorenansätze diskutiert wurden.

Eingeleitet wurde die Diskussion durch mehrere allgemeine Statements zu Funktion, Stellung und Bedeutung von Nachhaltigkeitsindikatoren. Folgende Indikatorenansätze wurden diskutiert:

In dem Projekt ,Zukunftsfähige Kommune - Wettbewerb und Kampagne zur Unterstützung der lokalen Agenda 21" der Deutschen Umwelthilfe testen 20 Pilotkommunen die in vier Leitkategorien eingeteilten 38 Hauptindikatoren, die um 14 optionale Indikatoren ergänzt werden können. Der Startschuss zur Datenerhebung fiel am 13. September 2001 und soll im März 2002 abgeschlossen werden.

Bei dem Projekt „Indikatoren für eine nachhaltige Entwicklung in NRW“ stellt das Land NRW den Kommunen ein Set von Basisindikatoren zur Verfügung. Diese Indikatoren werden in einer Pilotphase von Kommunen in NRW auf ihre Alltagstauglichkeit getestet. Die 28 Indikatoren kommen aus den Bereichen „Energie“, „Soziale Stadt“, „Stadtentwicklung und Ressourcen“" und "Stoffströme“, wobei jeweils generelle Indikatoren um einige ergänzende Indikatoren erweitert werden. Nach der zweijährigen Pilotphase sollen diese Indikatoren überprüft und bei Bedarf abgeändert oder ergänzt werden.

Das Indikatorensystem des Sonderforschungsbereiches SFB 419 „Umweltprobleme 
eines industriellen Ballungsraums: Naturwissenschaftliche Lösungsansätze und sozialwissenschaftliche Implikationen" an der Universität zu Köln hat, ausgehend von der häufig verwendet Einteilung der Nachhaltigkeitsproblematik in die drei Dimensionen Ökonomie, Soziales und Ökologie, zehn Themen ausgearbeitet, die von besonderer Bedeutung für die Nachhaltigkeit in NRW sein sollen. Während die ökonomische und die ökologische Dimension mit nahezu gleich vielen Themen vertreten sind, wird die soziale Dimension mit nur einem Themenfeld dargestellt. Den zehn Themenfeldern wurden insgesamt 28 Indikatoren zugeordnet, durch die die Nachhaltigkeit der jeweiligen Kommune in dem Themenfeld ermittelt wurde. Durch die Normierung der Skalen und die Bildung von Summenindices wurden Werte ermittelt, mit deren Hilfe die zwanzig beteiligten Regionen in Nachhaltigkeitsklassen zusammengefasst wurden. Charakteristika der einzelnen Regionen wurden u. a. mit Hilfe von Spinnendiagrammen dargestellt.

In der Kooperativen Indikatorenentwicklung im Märkischen Kreis wurden die Indikatoren von den Aktiven der Modellregion in sechs Handlungsfeldern erstellt. Für diese Handlungsfelder, wie z. B. regionale Vermarktung oder Verkehr, wurden Ziele aufgestellt, für die in einem weiteren Schritt Indikatoren vorgeschlagen wurden. Iterativ wurden diese Indikatoren reduziert, bis für jedes der Handlungsfelder ein Indikator übrig blieb. Auswahlkriterien für die Indikatoren waren Verständlichkeit, Datenverfügbarkeit und Beeinflussbarkeit.

Neben diesen vier Indikatorensystemen wurden von Beteiligten als Diskussionsbeiträge auch Beispiele aus anderen Indikatorensysteme angeführt.

Die Tagungsdokumentation ist erschienen in der Reihe Forschungsberichte aus dem SFB 419 der Universität zu Köln und kann unter http://www.uni-koeln.de/sfb419/ heruntergeladen werden.

\section{Internationaler Workshop „Ethik und Informationsgesell- schaft"}

\author{
Banská Bystrica, Slowakische Republik, 19. \\ April 2001
}

\section{Bericht von Daniela Fobelova und Monika Beköova, Matej Bel-Universität, Banská Bystrica}

\begin{abstract}
„Das 21. Jahrhundert wird ethisch sein oder es wird kein Jahrhundert mehr geben." (Gilles Lipovetsky)
\end{abstract}

Diese Prognose bezieht die ethische Diskussion auf neue Sphären und Bereiche: Bioethik, human orientiertes Verhalten ökonomischer Akteure, Umweltschutz, Ethik der Informationsgesellschaft usw. Die (Über-)Lebensfähigkeit (und damit auch das Risiko) der Ethik wird durch das Reflektieren neuer Probleme und damit auch durch das Verschwinden von der „guten alten Moral“" geprägt.

Ethik wird traditionell als philosophische Disziplin verstanden, die in die Sphäre der Philosophie gehört, die praktische Philosophie genannt wird. Diese Bezeichnung kommt ihr deswegen zu, weil zu ihren wichtigsten Aufgaben nicht (vorrangig) die theoretische Reflexion, sondern die Suche nach Antworten auf praktische Fragen unseres Lebens gehört. Philosophisch-ethische Forschung hätte keinen Sinn, wenn sie kein praxisbezogenes Denken wäre. Sie wäre lediglich sinnloses Moralisieren, wenn sich nicht gezeigt hätte, dass bzw. wie moralische Normen das Entscheiden und Handeln von Menschen beeinflussen. Die öffentliche Meinung, die einen untrennbaren Teil des gesellschaftlichen Lebens darstellt, ist auch mit Moral verbunden, und diese ist oftmals wirksamer als Gesetze. Heute spricht man von Ethik auch im Zusammenhang mit der Anwendung der Moral in spezifischen Bereichen der gesellschaftlichen Praxis. $\mathrm{Zu}$ den Aufgaben dieser angewandten Ethik gehört auch die ständige Bereicherung der Praxis einzelner Bereiche durch ethische Begriffe, Werte und auch praktisch-ethische Empfehlungen.

Vor diesem konzeptionellen Hintergrund hat am 19. April 2001 unter Leitung des Lehrstuhls Ethik und Ästhetik der Matej-Bel-Universität Banská Bystrica (Slowakische Repu- 